\title{
Los mecanismos de reforma a la constitución y la legitimación a los acuerdos de paz
}

\section{The mechanisms of constitutional reform in and the legitimation of the peace agreements}

\author{
José Luis Sañudo-Ospina ${ }^{1}$
}

\begin{abstract}
Resumen
El Estado colombiano se encuentra "ad portas" de cerrar uno de los capítulos más lamentables en su historia: el conflicto con las Fuerzas Armadas Revolucionarias de Colombia (FARC). Tras más de tres años de negociaciones, las partes han llegado a importantes acuerdos, destacándose el des-escalamiento del conflicto armado y consensos frente a mecanismos de justicia transicional. Con estos puntos claros, se da pie a que el Estado haga uso de los medios constitucionales y legales para la incorporación de los acuerdos de paz al ordenamiento jurídico nacional. El presidente Santos ha convocado al pueblo colombiano a la votación de un plebiscito que, para sorpresa de gran parte del electorado, no obtuvo el porcentaje necesario para refrendar los acuerdos de paz. Esto ha dejado a los mismos en una incertidumbre frente a la vía idónea para su aprobación, barajándose posibilidades como la legitimación ante el congreso, el cabildo abierto, y hasta un nuevo plebiscito. El objetivo de este artículo es analizar los principales mecanismos de reforma a la constitución que consagra el ordenamiento jurídico colombiano como medios legitimadores a los diálogos de paz. Plantearemos conceptos, regulación legal y constitucional, así como también semejanzas y diferencias existentes entre los mecanismos de participación ciudadana y reforma constitucional que pudiesen resultar apropiados para la materialización de los resultados esperados por el gobierno frente al proceso de paz.
\end{abstract}

Palabras clave: Acuerdos de paz; plebiscito; referendo cabildo abierto; congreso, participación ciudadana; reforma constitucional

\begin{abstract}
The Colombian state is about to close one of the worst chapters in its history: the war against FARC (Revolutionary Armed Forces of Colombia) that has lasted for more than five decades. After more than three years of discussions, the parties have settled on important agreements such as the de-escalation of the armed conflict and transitional justice mechanisms. It is thus time for the State to use constitutional and legal means to incorporate the agreements into the national legal system. President Juan Manuel Santos has called for a plebiscite as the means to legitimate

\author{
Tipología: Artículo de reflexión \\ Recibido: $28 / 03 / 2016$ \\ Evaluado: 03/11/2016 \\ Aceptado: 09/11/2016 \\ Disponible en línea: 01/03/2017 \\ Como citar este artículo: Sañudo-Ospina, J.L. (2017). Los mecanismos de reforma a la constitución y la legitimación a los acuerdos \\ de paz. Jangwa Pana, 16 (1), 103 -111. Doi: http://dx.doi.org/10.21676/16574923.1960
}

1. Abogado. Investigador en materia de Derechos Humanos. Grupo de Litigio Estratégico de la facultad de Derecho de la Universidad Pontificia Bolivariana. Colombia. Correo electrónico: joseluis.ea777@gmail.com. ORCID ID: 0000-0002-0354-2492 
the peace agreements. As a surprise to many, the plebiscite did not obtain the votes needed for its implementation, leaving it's future in uncertainty. The government is now considering other alternatives, such as pass it through congress, call for an open council meeting and even call for a new plebiscite. The main aim of this article is to analyze the mechanisms of constitutional reform existing in the Colombian legal system to validate the Havana Talks. Some concepts, and constitutional and legal regulations will be studied and various mechanisms of citizen participation and constitutional reform will be contrasted that may help to achieve the government expectations with the peace process.

Keywords: Colombian peace agreements; plebiscite; referendum; congress, open council meeting, citizen participation; constitutional reform

\section{Introducción}

$\mathrm{L}$ a razón de ser de esta investigación, surge a raíz del posible cierre de un acuerdo entre gobierno y FARC en materia de paz cuyo objetivo es poner fin a un conflicto armado que supera ya las cinco décadas (Zuleta, 1991). En este orden de ideas, el gobierno colombiano anunció que el medio para la inclusión de dichos acuerdos al ordenamiento jurídico colombiano sería el plebiscito. El pueblo, por medio del ejercicio del voto, se pronunció, arrojando como resultado la reprobación de lo dialogado y dejando en un limbo el acuerdo definitivo de paz. A raíz de tales acontecimientos, el gobierno colombiano se ha planteado varias opciones para la implementación de los acuerdos de paz, que se supone serán resultado de conciliaciones previas con la oposición.

Surge, entonces, el interrogante acerca de las vías adecuadas para la refrendación de los acuerdos, teniendo en cuenta que el ordenamiento jurídico colombiano consagra otros medios distintos al plebiscito para la reforma a la constitución que talvez pudiesen resultar más adecuados a las necesidades que se presentan en la actualidad dentro de la sociedad colombiana. Inquietud que intentaremos resolver a través de esta investigación, la cual abordaremos de la siguiente manera (Registraduría Nacional del Estado Civil, 2016):
En primer lugar, realizaremos una breve síntesis sobre las raíces del conflicto, los acercamientos de paz previos y el desarrollo de los diálogos en Cuba. En segunda instancia, entraremos a estudiar los mecanismos de reforma a la Constitución consagrados en el ordenamiento jurídico colombiano, los conceptos establecidos por la ley, la doctrina y la jurisprudencia, así como sus semejanzas y diferencias (Sierra, 2009). Posteriormente, explicaremos por qué es inviable el medio de refrendación que propone la guerrilla. De igual modo analizaremos la aplicación de los mecanismos mencionados anteriormente en relación con la situación actual de la sociedad colombiana. Finalmente, entraremos a revisar las conclusiones obtenidas de esta labor.

\section{Origen del conflicto y acercamientos de paz previos}

La violencia política y la defensa de territorios rurales provocan la formación de movimientos armados campesinos; movimientos que, en su constante pugna por la lucha de sus derechos contra el gobierno, terminan dispersándose por las montañas colombianas y conformando Las Fuerzas Armadas Revolucionarias de Colombia, Ejército del Pueblo (FARC-EP): el movimiento guerrillero más antiguo del mundo (Cadavid, 2010). 
Durante las más de cinco décadas que ha durado el conflicto, han sido varios los acercamientos intentados por el gobierno hacia una eventual pazcon la insurgencia armada. Entre dichos acercamientos encontramos, por ejemplo, "Los diálogos de La Uribe", en el departamento del Meta: se trató de conversaciones impulsadas en los años 80 por la administración de Belisario Betancur que, a pesar de arrojar acuerdos, fracasaron por la persecución de grupos paramilitares a la Unión Patriótica (brazo político de las FARC) y por las actividades ilícitas de algunos sectores de la guerrilla que se opusieron a la entrega de las armas (Centro de Memoria, Paz y Reconciliación, 2014). A finales de la década de los 90, el presidente Andrés Pastrana inicia unos nuevos acercamientos con miras a concretar el final del conflicto con la guerrilla. Tales acercamientos fueron ampliamente cuestionados por un gran sector de la sociedad colombiana, en razón a las concesiones que el gobierno otorgó al grupo insurgente. La llamada "Zona de despeje", por citar un caso concreto, fue un territorio libre de presencia militar donde las FARC impusieron su ley y orden, facilitando la realización de actividades ilícitas como el narcotráfico, secuestro y atentados terroristas. Hechos como este provocarían, a la postre, el rompimiento unilateral de los diálogos por parte del gobierno, y el fin de los acercamientos de paz durante una década (Centro de Memoria, Paz y Reconciliación, 2014).

\section{El proceso de paz en La Habana}

En agosto del 2012, se hicieron públicos los acercamientos que el gobierno de Juan Manuel Santos venía adelantando con las FARC. Para garantizar la transparencia de los diálogos y evitar repetir circunstancias del pasado como las que llevaron al fracaso de las conversaciones durante el gobierno de Pastrana, se acordó que los diálogos se desarrollarían en un Estado ajeno, resultando Cuba el lugar acordado para los mismos.

Después de más de tres años de conversaciones con puntos álgidos y momentos de tensión que pusieron en riesgo la estabilidad de las mismas, en Septiembre del 2015, dentro de un encuentro sin precedentes, el primer mandatario de los colombianos se reunió con Timoleón Jiménez (Timochenko), máximo comandante de las FARC, para la firma de un principio de acuerdo en materia de víctimas y justicia (Semana, 2015). Seguidamente a este encuentro, el presidente Santos anunció su intención de incorporar los acuerdos de paz al ordenamiento jurídico colombiano por medio de un plebiscito. Sin embargo, el grupo insurgente, a través de comunicaciones, ha manifestado expresamente que no considera este mecanismo como el medio adecuado de convalidar los acuerdos de paz y que, por el contrario, considera necesario convocar a una asamblea nacional constituyente para que se promulgue una nueva carta política que incluya lo acordado en Cuba:

\section{No aceptamos la aplicación del llamado plebiscito para refrendación del acuerdo final [porque ese pacto] que traerá la paz a Colombia, que se está construyendo con tanto esmero y sacrificio, no puede ser puesto en peligro por ligerezas políticas (Vanguardia, 2016).}

Frente a las anteriores declaraciones, Santos respondió a las FARC defendiendo su posición en cuanto a la viabilidad del plebiscito afirmando que: "es un mecanismo democrático por medio del cual el pueblo colombiano puede pronunciarse si aprueba o no los acuerdos de paz" (Semana, 2015). De igual modo, negó rotundamente la posibilidad de convocar a una asamblea nacional constituyente para la refrendación de los acuerdos.

El pasado mes de octubre, el pueblo como constituyente primario se manifestó frente a la refrendación de los acuerdos de paz acudiendo a la votación del mencionado plebiscito. Sin embargo, con un sorprende resultado y por un corto margen de diferencia la oposición salió avante y los acuerdos de paz se han visto en vilo desde entonces. A raíz de lo anterior, la administración del presidente Santos ha convocado a la oposición para replantear los acuerdos de paz y así conciliar las divergencias que provocaron el rechazo de lo acordado. No obstante, los negociadores de las FARC no han visto con buenos ojos la intervención del senador 
Uribe Vélez en una eventual renegociación, y se mantienen firmes en la idea de la implementación de los acuerdos tal y como se pactaron en principio (El Correo, 2016).

Lo anterior da pie entonces para abrir varios interrogantes: ¿Es el plebiscito la vía jurídica adecuada para legitimar lo pactado?, ¿La idea que plantean Las FARC podría dar una mayor inclusión a las discusiones en la Habana?, o bien, ¿dentro de los mecanismos de reforma a la constitución y participación democrática que ofrece el ordenamiento jurídico colombiano, puede existir una modalidad más idónea para estos fines?

\section{Los mecanismos de participación popular y la reforma constitucional en Colombia}

Antes de abordar la discusión que suscitan los temas tratados en este artículo, es preciso delimitar ciertos conceptos necesarios para una mejor compresión de la inquietud a debatir. En primer lugar, debemos preguntarnos sobre el concepto de "mecanismos de participación democrática" o "participación ciudadana". El profesor Jacobo Pérez Escobar (1997) en su obra Derecho Constitucional Colombiano ofrece la siguiente definición "Los mecanismos de participación democrática son precisamente los adecuados para que la soberanía popular se ejerza tanto en el aspecto político como en lo que se refiere a la administración de los asuntos públicos" (p.101).

Así pues, considerando la anterior definición, y para efectos de esta investigación, podemos entender que los mecanismos de participación democrática son los medios idóneos para la materialización de la voluntad popular. Materialización que se ve reflejada en un cambio al orden jurídico interno de un Estado a raíz de una modificación constitucional producida por dichos mecanismos. ¿Y cuáles son los efectos de dicha modificación? Podríamos afirmar que estos se constituyen en la transformación de aspectos relevantes en materia política, social, económica y cultural dentro de un Estado determinado en el marco de las vías jurídicas y legales consagradas en el orden interno para tales fines.

En este orden de ideas, la constitución colombiana consagra varios mecanismos de participación democrática, cuyos efectos y aplicación en el ordenamiento jurídico dependerán del motivo para los cuales sean empleados y el ente u organismo competente para llevar a cabo los mismos. Así pues, la carta política de 1991, en su artículo 374, prescribe los mecanismos de reforma a la constitución, los cuales encuentran una regulación más amplia y uniforme en la ley estatutaria que regula los mecanismos de participación ciudadana, tal y como veremos a continuación.

\section{Conceptos de "referendo" y "plebiscito", y su aplicabilidad}

La ley estatutaria 134 de 1994, regula la ejecución de los mecanismos de participación democrática, entre los cuales se encuentran definidos el referendo y el plebiscito, en los artículos tercero y séptimo. Frente al primero se refiere de la siguiente manera: "El referendo es la convocatoria que se hace al pueblo para que apruebe o rechace un proyecto de norma jurídica o derogue o no una norma ya vigente". Podemos afirmar, entonces, que el referendo es el medio por el cual el constituyente primario se pronuncia bien sobre la viabilidad constitucional de una o más normas jurídicas preexistentes en el ordenamiento, bien sobre la posibilidad de incluir nuevas disposiciones que sean acordes a la normatividad interna de un Estado. Referenciando a Francisco Gómez Sierra (2009) en la constitución:

\begin{abstract}
El referendo es el mecanismo mediante el cual el pueblo aprueba o rechaza las decisiones normativas de las autoridades, expresadas en un texto ya elaborado de proyecto; lo hay para derogar reformas constitucionales y leyes, para aprobar reformas a la constitución (p.209).
\end{abstract}

Frente al plebiscito, la ley citada anteriormente considera lo siguiente en su artículo séptimo: "El plebiscito es el pronunciamiento del pueblo convocado por el presidente de la república mediante el cual apoya o rechaza una determinada 
decisión del ejecutivo". Consideremos entonces que el plebiscito es el llamado que hace la cabeza del gobierno a la población para que manifiesten su aprobación u oposición frente a una iniciativa que esta pretende promover. La Corte Constitucional en sentencia C-180 del 14 de Abril de 1994, se pronuncia sobre la constitucionalidad del plebiscito, y precisa que para que este pueda llevarse a cabo debe cumplir con los siguientes lineamientos: "El presidente de la República con la firma de todos los ministros y previo concepto favorable del Senado de la República, podrá consultar al pueblo decisiones de trascendencia nacional. La decisión del pueblo será obligatoria" Podemos afirmar entonces que lo pretendido por el supremo tribunal constitucional colombiano con esa sentencia fue, en primer lugar, hacer hincapié en que la iniciativa contenida en el plebiscito debe tener el aval del gabinete ministerial del presidente. Esto quiere decir que no puede interpretarse como una iniciativa exclusiva del jefe de gobierno, sino que debe contar con el apoyo de sus colaboradores, en una manifestación conjunta de propuesta única por parte del ejecutivo. En segundo lugar, al referirse al "concepto previo y favorable del Senado de la República", el tribunal hace constar que es indispensable la revisión de la iniciativa por parte de una de las cámaras del congreso, en virtud de garantizar el principio de control interinstitucional que deben ejercer las ramas del poder público entre sí, así como de revisar la legalidad de la misma y evitar una extralimitación del ejecutivo en sus facultades.

Delimitados ya los conceptos de "referendo" y "plebiscito", notamos que en principio pueden parecer bastante similares ya que la finalidad de los dos se sintetiza en una reforma constitucional: "El procedimiento en uno y otro es semejante en cuanto ambos operan por el voto directo del cuerpo electoral con un sí o un no sobre una pregunta o un proyecto de decisión que le es sometida a consideración". Sin embargo, vale la pena señalar que existen ciertas diferencias que la doctrina se ha encargado de distinguir. El jurista Paolo Biscaretti di Ruffia (1982), por ejemplo, hace la siguiente distinción:
A pesar de que la doctrina y la legislación frecuentemente usan indistintamente, los términos de referendo y plebiscito, este último (siempre caracterizado por una nota de excepcionalidad y de carácter extraordinario, que ha excluido hasta ahora cualquier regulación suya uniforme, de orden tanto interno como internacional), debería más precisamente referirse a una manifestación del cuerpo electoral no actuada en relación a un acto normativo (como el referendo), sino más bien, respecto a un simple hecho o suceso, concerniente a la estructura esencial del Estado o de su gobierno (por ejemplo, una adjudicación de territorio, el mantenimiento o la mutación, de una forma de gobierno, la designación de una determinada persona en un oficio particular, etc.) (p.421).

Elautoritalianoconsideracomocriteriodiferenciador de ambos conceptos la "extraordinariedad"; es decir que el jurista europeo considera el plebiscito como algo que debe aplicarse en situaciones extrañas, y fuera de lo común a la normalidad del ámbito estatal, que deben ser resueltas a través de la decisión del pueblo. Mientras que el referendo se limita a la resolución de una controversia contenida dentro de una norma perteneciente al ordenamiento jurídico interno de los Estados.

Complementando lo expuesto anteriormente, con lo expresado por el ya mencionado autor nacional Pérez Escobar (1997), de esta manera:

En cambio, como hemos visto, el referendo tiene únicamente por objeto que el pueblo o el cuerpo electoral directamente apruebe o rechace un proyecto de norma jurídica o derogue o no una norma ya vigente. Esto es que el referendo siempre tiene como contenido una norma jurídica que se proyecta expedir o una ya vigente. Por lo mismo, le son ajenos como objeto de decisión los simples hechos y sucesos (p.108).

Para concluir, podemos afirmar entonces que el principal factor diferenciador entre un concepto $\mathrm{y}$ el otro radica en el objeto susceptible de 
modificación; es decir que si, por un lado, lo que se buscar es otorgar solución a una circunstancia imprevista o poco común dentro del Estado, lo idóneo sería la aplicación del plebiscito, y que si, por otro lado, lo que se busca es añadir o remover una norma contemplada en la normatividad vigente de este, el mecanismo adecuado a utilizar sería el referendo. Teorizados y diferenciados estos conceptos, pasemos ahora a estudiar la viabilidad de uno u otro frente al contexto de la eventual inclusión de los acuerdos de paz en Colombia.

\section{La inviabilidad de una constituyente}

Tal y como mencionamos previamente, a raíz del rechazo del plebiscito, los diálogos de paz se encuentran en un limbo cuya resolución aún es incierta. Lo anterior, ha suscitado la posibilidad de convocar a una Asamblea Nacional Constituyente como vía de adhesión a los acuerdos, tal y como lo propusiese las FARC durante los diálogos.

A pesar de que el gobierno ya ha afirmado rotundamente que no convocará a una asamblea nacional constituyente, vale la pena hacer un análisis de por qué es inconveniente esta vía que propone las FARC para las instituciones jurídicas del Estado colombiano. El artículo 376 de la Carta consagra el concepto de "Asamblea Nacional Constituyente"; un concepto que el jurista colombiano Vladimiro Naranjo Mesa en su obra Teoría Constitucional e Instituciones Políticas (2010) explica de la siguiente manera:

Una asamblea nacional constituyente es una corporación especialmente integrada para elaborar un texto constitucional para un Estado en formación, para reemplazar una Constitución ya existente, o para introducirle a las vigentes modificaciones sustanciales, básicamente en cuanto a la forma del Estado, al sistema de gobierno, o al régimen político (p.384)

De lo prescrito anteriormente podemos afirmar que este es el mecanismo de reforma constitucional más radical, pues el resultado del mismo no es precisamente una modificación a las normas, sino el nacimiento de un nuevo orden constitucional.

Así pues, y para argumentar por qué no es conveniente la propuesta de las FARC de una asamblea constituyente, debemos remitirnos a los principios constitucionales, los cuales podemos catalogar como aquellos axiomas fundamentales sobre los cuales debe sostenerse cualquier constitución. Dentro de estos, hallamos el "Principio de Estabilidad Constitucional", del cual se desprende la idea de que las constituciones deben estar diseñadas para perdurar en el tiempo, pues estas se levantan como el origen y fundamento del ordenamiento jurídico de un Estado. Con base a lo anterior, y teniendo en cuenta que la Constitución de 1991 es una constitución bastante joven comparada con otras constituciones como la norteamericana (la cual se encuentra vigente desde 1787) o la anterior carta colombiana (que fue usada durante más de cien años), consideramos que no es conveniente un cambio de constitución como justificación a los acuerdos de paz, pues las instituciones concebidas bajo un orden constitucional deben entenderse en un sentido permanente y duradero para garantizar la estabilidad del ordenamiento jurídico interno de los Estados.

\section{La vía adecuada de legitimación}

Es momento de entrar a analizar debidamente la inquietud que ha llevado a la realización de esta investigación, la cual es determinar cuál es el mecanismo adecuado para refrendar las negociaciones de paz en Cuba. Anteriormente brindamos nuestro argumento sobre por qué no es conveniente la realización de una asamblea nacional constituyente, tal y como lo propone Las FARC. Es necesario entonces, y ya explicados los conceptos de "plebiscito" y "referendo", entrar a observar cuál de estos mecanismos es viable para poder materializar los resultados de casi cuatro años de negociaciones acorde al panorama colombiano en la actualidad considerando, como lo mencionamos anteriormente, que la propuesta de refrendar los acuerdos a través de un plebiscito 
fracasó y que, por lo tanto, el gobierno considera ahora otros medios sin descartar la realización de un nuevo acto plebiscitario.

Con respecto al referendo, consideramos que el trato dado a este mecanismo de reforma en Colombia se limita a legitimar decisiones estructurales dentro de la rama ejecutiva, tales como la reelección presidencial, la cual fue promovida y aprobada durante el primer mandato de Álvaro Uribe Vélez. Por lo anterior, y teniendo en cuenta que lo que se busca con los acuerdos de paz es una desmovilización del brazo armado de la insurgencia, lo cual necesariamente ha de incluir medios y mecanismos de reinserción que aseguren no solo la incorporación exitosa de los desmovilizados a la sociedad civil, sino también garantías a la estabilidad y tranquilidad de la sociedad. Es por esto que, a nuestro criterio, consideramos que el referendo se queda corto frente a las pretensiones que se tienen con la firma de un acuerdo de paz definitivo, pues dentro de sus alcances no está el brindar las herramientas necesarias para una verdadera modificación del panorama social colombiano: punto esencial en la materialización de los acuerdos de paz.

Si bien el plebiscito fue presentado como el medio más adecuado para legitimar los acuerdos según los objetivos del gobierno, consideramos que este también se quedaba corto en los términos planteados por la administración de Santos. A pesar de que, según los negociadores de parte y parte, ya existían acuerdos en materia de justicia transicional y víctimas, aún no se ha sido muy claro frente a los medios y mecanismos de reinserción social que va a implementar el gobierno con los insurgentes que abandonen las armas. Aunque, uno de los ejes de este artículo no es precisamente el tema de justicia transicional, vale la pena invocar la definición que propone el profesor Rodrigo Uprimny (2005) para ilustrar un poco el tema: "La justicia transicional [dice] hace referencia a aquellos procesos transicionales mediante los cuales se llevan a cabo transformaciones radicales de un orden social y político determinado, que enfrentan la necesidad de equilibrar las exigencias de paz y justicia”.
A partir de lo enunciado con anterioridad, podemos afirmar que la principal característica de la justicia de transición es su “extraordinariedad”, ya que como bien lo menciona el profesor Uprimny es el medio para restablecer la armonía de una sociedad que ha sufrido una grave alteración a sus instituciones y al orden público. Ese punto en particular fue bastante controvertido por parte de quienes se opusieron al plebiscito, pues consideraban que los desmovilizados debían someterse a la justicia ordinaria, pues unos tribunales de justicia transicional podrían generar impunidad, impidiendo así la realización de la verdad, justicia y reparación que son los tres principios fundamentales de la justicia transicional.

Así pues, y regresando al por qué el plebiscito no ofrecía una verdadera solución a lo que se buscaba con los acuerdos de paz, encontramos que este no se compadecía con las necesidades que presenta la sociedad colombiana en la actualidad. Por tanto, el resultado de esta consulta no materializaría como tal la voluntad real del pueblo. Citando a Hans Kelsen en su obra Teoría Pura del Derecho (1960) encontramos:

El desarrollo legal de disposiciones constitucionales debe guardar una estrecha concordancia con el carácter progresivo de los derechos enunciados en la Carta Política, razón por la cual, no basta con regular los mecanismos de participación democrática, debe garantizarse que estos mecanismos materialicen realmente el querer de la ciudadanía y así lo puedan expresar en un pronunciamiento popular (p.118).

De acuerdo con esto, el gobierno debe plantear una nueva vía que se adecue realmente a las circunstancias que atraviesa la sociedad colombiana en la actualidad. Vale la pena recordar que los mecanismos implementados deben mirar no solo hacia el presente, sino tener en cuenta también las eventualidades que puedan surgir en un futuro, pues si bien el fin del proceso se verá enmarcado con la legitimación de los acuerdos, este marcará el inicio de lo que podría ser una verdadera construcción de paz. 
En este orden de ideas, la discusión frente a la idoneidad de los mecanismos adecuados para refrendar los acuerdos de paz permanecerá abierta, a raíz del ya mencionado resultado del plebiscito. El presidente Santos ha anunciado que se barajan las posibilidades de refrendar los acuerdos vía congreso, cabildo abierto, o hasta a través de un nuevo plebiscito.

La opción de tramitar los acuerdos por medio del congreso, a través de un acto legislativo, era considerada por muchos como la vía que se debió implementar desde el principio, pues ofrecía el escenario idóneo para la revisión y discusión de lo discutido en Cuba por las distintas fuerzas políticas que apoyaban y se oponían al mismo. Sin embargo, la administración consideró que era el pueblo a través del voto y el plebiscito quien debía tener la última palabra.

De igual modo, se ha estudiado la propuesta de un cabildo abierto, el cual está regulado en la ley 134 de 1994, y el que es definido en la misma así:

El Cabildo abierto es la reunión pública de los concejos distritales, municipales o de las juntas administradoras locales, en la cual los habitantes pueden participar directamente con el fin de discutir asuntos de interés para la comunidad (Colombia, Congreso de la República de, 1994).

Frente a esto, es difícil hacer un análisis de conveniencia dado que los antecedentes de refrendación de algo de tal magnitud e importancia para la sociedad colombiana como lo son los acuerdos de paz, a través de este mecanismo, son prácticamente nulos.

Finalmente, la última opción que se maneja es la de convocar a un nuevo plebiscito; sin embargo, consideramos que esta opción es totalmente inviable dado el gasto que implica para el Estado realizar una jornada electoral extraordinaria. Además, nada garantiza que por medio de un nuevo plebiscito pueda obtenerse un resultado mayoritariamente favorable que refleje la aprobación general del pueblo frente al acuerdo re-negociado.

\section{Conclusiones}

Para finalizar esta investigación, podemos concluir lo siguiente:

Si bien no es la primera vez que se da un intento de llegar a un acuerdo de paz con las FARC-EP, este proceso ha materializado una verdadera voluntad de terminar el conflicto por parte y parte. Aunque la iniciativa plebiscitaria rechazó lo acordado en principio, tanto el gobierno como las FARC han mostrado una voluntad de no desechar las negociaciones. De igual modo, en un intento por conciliar posiciones, el gobierno ha invitado a la oposición a participar activamente en la reforma de los acuerdos. Dicha reforma será llevada a Cuba para que sea estudiada en conjunto por las delegaciones de la guerrilla y del gobierno, y para que posteriormente sea posible definir un medio definitivo de legitimación.

En segunda instancia, a través de esta investigación se ha realizado una distinción conceptual entre los mecanismos de reforma constitucional. Según nuestro criterio, hemos brindado un análisis que parte del qué tan conveniente es aplicar un mecanismo o el otro, apoyados para esto en la legislación colombiana, la doctrina y los pronunciamientos judiciales respectivos.

La conclusión final a la que podemos llegar es que, si bien el plebiscito pudo no haber sido un medio adecuado para materializar los efectos del proceso de paz, el gobierno debe plantear una forma adecuada para la ejecución del mismo. Considerando además que aún no es muy claro cuáles serán los medios por los cuales la administración llevará a cabo el desescalamiento del conflicto armado y la reinserción de los desmovilizados a la sociedad civil.

Al concluir esta investigación encontramos que ha surgido una nueva propuesta de legitimación para los acuerdos de paz, que eventualmente podría sumarse a las alternativas mencionadas con anterioridad. Esta propuesta se basa en las disposiciones consagradas por los Convenios y protocolos de Ginebra, cuerpos de normatividad internacional que regulan todo 
lo referente al Derecho de la Guerra y el Derecho Internacional Humanitario. Se propone que, de llegarse a un pacto sostenido en estos tratados, los mismos serían incluidos al ordenamiento jurídico colombiano por medio del bloque de constitucionalidad, sin necesidad de llevarse a cabo un plebiscito (Semana, 2016) .

Sea cual sea la decisión final sobre el mecanismo de refrendación, esperamos que esto implique un valioso aporte a la sociedad colombiana y que, de igual modo, contribuya a alcanzar el fin definitivo de uno de los conflictos internos más duraderos en la historia reciente.

\section{Referencias bibliográficas}

Cadavid, E.S. (2010). Historia de la guerrilla en Colombia. Recuperado de http://ecsbdefesa.com.br/: http://ecsbdefesa.com.br/defesa/fts/HGC.pdf

Centro de Memoria, Paz y Reconciliación. (2014). Los Pactos de la Uribe FARC- EP (1984 y 1986) y Dialogo Nacional con el M19, EPL y ADO (1984). Bogotá D.C: Alcaldía Mayor de Bogotá. Recuperado de http://centromemoria.gov.co/:http://centromemoria.gov.co/wp-content/uploads/2014/03/Los-Pactosde-la-Uribe-FARC-EP-1984-y-1986-y-DialogoNacional-con-el-M19-EPL-y-ADO-1984.pdf

Colombia. (1994). Corte Constitucional. Sentencia C-180 de 1994. Democracia participativa-alcance/ Participación ciudadana.

Colombia. (mayo 31 de 1994). Congreso de la República. Ley 134 de 1994, por la cual se dictan normas sobre mecanismos de participación ciudadana.

Escobar, J. P. (1997). Derecho Constitucional Colombiano. Bogotá: Temis.

Kelsen, H. (1960). Teoría Pura del Derecho. Buenos Aires: Editorial Universitaria de Buenos Aires.

Mesa, V. N. (2010). Teoría Constitucional e Instituciones Políticas. Bogotá: Temis.
Política. (2016, 23 de febrero). Lo de la Guajira "no fue un pequeño impase": Comisionado de paz. El Tiempo. Recuperado de http:// www.eltiempo.com/politica/proceso-de-paz/ miembros-de-las-farc-en-la-guajira/16518819

Registraduría Nacional del Estado Civil. (2016). Referendo, consulta, plebiscito o constituyente: las vías para refrendar la paz. Mecanismos de participación ciudadana. Bogotá D.C: Registraduría Nacional del Estado Civil. Recuperado de http://www.registraduria.gov.co/Referendo-consulta-plebiscito-o.html

Ruffia, P. B. (1982). Las Instituciones Legislativas de la Democracia Directa. Madrid: Tecnos.

Semana. (2015, 12 de noviembre). Santos defiende plebiscito para refrendar acuerdo de paz. Revista Semana. Recuperado de http://www.semana.com/nacion/articulo/santos-defiende-plebiscito-para-refrendar-acuerdo-de-paz/449612-3

Semana. (2015, 23 de septiembre). Gran encuentro de Santos, 'Timochenko' y Raúl Castro. Revista Semana. Recuperado de http://www.semana.com/nacion/articulo/santos-defiende-plebiscito-para-refrendar-acuerdo-de-paz/449612-3

Semana. (2016, 19 de marzo). ¿Un acuerdo especial en lugar de refrendación? Revista Semana. Recuperado de http://www.semana.com/nacion/articulo/acuerdoespecial-podria-sustituir-refrendacion-de-acuerdosde-paz/466005

Sierra, F. G. (2009). Constitución Política de Colombia Anotada. Bogotá: Leyer.

Vanguardia. (2016). Santos, firme con el plebiscito "les guste o no a las Farc". Colombia Vanguardia: Resumen de agencias. Recuperado de http://www. vanguardia.com/colombia/346596-santos-firme-conel-plebiscito-les-guste-o-no-a-las-farc

Zuleta, E. (1991). Colombia: Violencia, Democracia y Derechos Humanos. Bogotá: Altamir. 